\title{
ICT in Sanità: vecchi problemi e nuove sfide
}

\section{G. Quintaliani ${ }^{1}$, R. Torpia ${ }^{2}$, M. Giovannelli ${ }^{3}$, A. Orecchini ${ }^{4}$, L. De Angelis ${ }^{4}$}

${ }^{1}$ S.C. Nefrologia e Dialisi, Azienda Ospedaliera di Perugia, Perugia

${ }^{2}$ S.C. Nefrologia e Dialisi, Azienda Ospedaliera, Ospedale di Circolo, Busto Arsizio

${ }^{3}$ CUP, Azienda Ospedaliera di Perugia, Perugia

${ }^{4}$ Laboratorio Analisi, Azienda Ospedale Perugia, Perugia

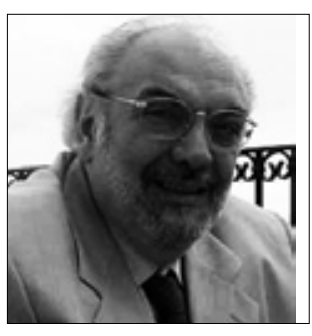

Giuseppe Quintaliani
Recentemente un articolo del Sole24 Ore Sanità (1) basato su una ricerca condotta dall'Osservatorio Ict\&Cio in Sanità della School of Management del Politecnico di Milano, apriva con queste parole: «'̀ sempre più evidente, in tutti i settori, il ruolo strategico delle tecnologie ICT (Information\& Comunication Technology) (2), in grado non solo di migliorare l'efficienza, ma anche di differenziare la qualità dei servizi erogati». Inoltre veniva sottolineata la crescente consapevolezza che «l'ICT sia una valida leva di cambiamento, la cui strategicità aumenta al crescere delle trasformazioni organizzative in atto». Nel campo della Sanità, lo stesso articolo individuava i fondamentali temi per i quali la tecnologia ICT è oggetto di crescente interesse applicativo:

- la razionalizzazione dei processi gestionali e amministrativi permettendo di garantire la corretta raccolta ed elaborazione dei dati che devono essere trasmessi agli organi istituzionali e di snellire le procedure burocratiche che caratterizzano il settore;

- il miglioramento della qualità dei processi clinici e sanitari contribuendo a migliorare la qualità della cura erogata e a ridurre il rischio clinico;

- la continuità assistenziale, consentendo l'integrazione di tutti gli enti che concorrono sul territorio all'erogazione della prestazione clinica.

Dall'analisi di 100 strutture sanitarie italiane, oggetto della ricerca, emergeva che le priorità di investimento nelle tecnologie ICT sono orientate ai processi primari di cura nel 65\% dei casi, di diagnosi nel 33\%, seguiti dai processi di supporto relativi alla programmazione e controllo (29\%) e qualità e rischio (26\%). Tuttavia non venivano specificati quali processi di cura e diagnosi si avvalgono dell'informatica al servizio della pratica clinica. Citando l'uso che se ne fa della ICT, ad esempio per la consultazione di referti in ambito radiologico o dei laboratori analisi, possiamo ben dire che il valore aggiunto è importante ma non dirimente. Come appare ovvio, ben altro valore rappresenterebbe la possibilità di poter usufruire dell'elaborazione dei dati resi disponibili: in altre parole un uso della tecnologia informatica finalizzato al miglioramento dell'efficienza e qualità di cura. Ricordiamo che il paziente deve essere messo al centro del sistema (come recitano le norme della certificazione ISO), pertanto qualunque ICT deve essere concepita prioritariamente come utile al paziente, mediante sue applicazione in tutti i processi che conducono all'aumento di efficienza e qualità delle cure, e alla riduzione di errori e sprechi. Tutto ciò non può però prescindere da un profondo cambiamento culturale e organizzativo attraverso la partecipazione di tutti gli attori che concorrono ai processi di cure. Che cosa dunque vorremmo ottenere dall'uso della ICT in Sanità?

\section{Un sistema capace di snellire atti burocratici ed eliminare ripetitività e ridondanze}

La prima cosa di cui vorrebbero disporre i sanitari è un sistema affidabile che permetta di migliorare il modo di lavorare rendendolo più efficace e sicuro, in grado inoltre di omettere tutto ciò che, ripetitivo, burocratico $\mathrm{e}$ ridondante, sottrae del tempo alla cura del paziente.

Un sistema affidabile permetterebbe di dare spazio e concretezza al concetto di ufficio senza carta (pochi sanno che il decreto Brunetta sull'assenteismo, che così tanto ha fatto parlare di sé, conteneva anche il concetto di ufficio senza carta). 
Si parla spesso di cartella clinica informatizzata, sentita come strumento fortemente innovativo. Molte volte concepita come comune contenitore di informazioni e dati clinici, la cartella clinica così congeniata, apporta certamente il vantaggio della bella calligrafia (criterio forte per la difficile leggibilità di certe grafie), della consultazione da diverse postazioni di lavoro (criterio debole poiché la maggior parte delle volte viene consultata al letto del malato o sale medici e infermieri), della facilitazione nella stesura della lettera di dimissione (criterio medio in quanto mera operazione di copia-incolla). Molte strutture si sono dotate di cartelle cliniche computerizzate, spesso di tipo "aziendale" non differenziate per tipologia di pazienti e/o gruppi di patologie. Ma la vera forza innovativa di una cartella clinica computerizzata, come esemplificato da alcune già esistenti per la specifica gestione dei pazienti uremici in dialisi e per gli ambulatori di nefrologia, è rappresentata dalla possibilità di valutare il trend del singolo paziente, elaborare i dati clinici e operare correlazioni (ad esempio, PA e terapia farmacologica, $\mathrm{Kt} / \mathrm{V}$ e stato nutrizionale ecc.) oltre alla possibilità di raggruppare pazienti sotto diverse caratteristiche (peso, età, sesso, parametro clinico ecc.). La vera forza innovativa di una cartella clinica informatizzata è infine la possibilità di poter valutare i target di reparto mediante l'analisi d'insieme di gruppi di pazienti. Ebbene, molte volte una cartella clinica elettronica aziendale, non permette di ottenere né il trend, né il raggruppamento dei pazienti per classi. Molto spesso non è neppure resa possibile l'estrazione dei dati per ottenere un database elaborabile. Qualunque sistema informatico veramente utilizzabile potrebbe registrare immediatamente lo scostamento e la probabilità di errore (ad esempio, il calcolo della probabilità di rigetto di trapianto renale mediante l'applicazione del teorema di Bayes [3]), potrebbe fornire il trend di qualunque parametro di valutazione clinica del paziente, stimare l'andamento nel tempo di parametri clinici e biochimici, fornire supporti esperti che aiutino nella diagnosi e terapia e soprattutto fornire previsioni. Ripensando ai pazienti nefropatici seguiti nei nostri ambulatori, è immediatamente immaginabile l'utilità di un sistema in grado di darci informazioni, ad esempio, sullo "slope" del reciproco della creatinina, sull'andamento del filtrato glomerulare, sul valore di emoglobina in correlazione alla somministrazione di eritropoietina ecc.

Il problema della ICT non si esaurisce tuttavia con la cartella clinica, tutt'altro! Ciò che oggi più assilla i sanitari, medici e infermieri, è la ripetitività ossessiva di alcune procedure. Un valido esempio è rappresentato dalla numerosità di moduli che, diversamente formulati, debbono essere compilati per la richiesta di ogni indagine radiologica, strumentale, visite e consulenze, farma- ci, trasfusioni di sangue ecc. Senza dimenticare che in alcune realtà, per "tracciare il prodotto cartella clinica" viene richiesto che ciascun foglio riporti del paziente il nome e cognome, la data di nascita e talvolta anche il numero SDO. Sarebbe semplificante poter stampare modelli di richieste comuni, adottando form omogenei, almeno per i dati anagrafici attinti da "database" aziendali (ad esempio, quello dei ricoveri) esistenti ma quasi mai disponibili.

Continuando con le esemplificazioni, l'informatizzazione della richiesta trasfusionale, ridurrebbe i tempi di consegna e la possibilità di potenziali errori. Liter prevede dapprima la compilazione di due diversi form, uno per la richiesta di sangue e un altro per la richiesta del gruppo sanguigno: la compilazione manuale è foriera di potenziali errori di trascrizione dei dati identificativi del paziente mentre una procedura informatica $a d$ hoc permetterebbe sempre di verificare la correttezza nell'immissione del dato, snellendo inoltre il sistema delle richieste. Continuando, la procedura richiede che il medico, ulteriormente verificata l'identità del paziente, confermi la congruità delle sacche inviate dal centro trasfusionale mediante la comparazione di serie numeriche. Si tenga conto che questo iter viene seguito, ad esempio, nel corso della guardia interdivisionale caratterizzata da una notevole e non programmabile mole di attività clinica, in reparti di degenza diversamente dislocati in vaste aree ospedaliere: un qualunque sistema per lettura di codice a barre (come quelle in uso presso le casse dei supermercati!) sarebbe in grado di evidenziare con assoluta sicurezza, la corrispondenza di due serie numeriche mentre l'apposizione della firma, a conferma della responsabile autorizzazione alla trasfusione, potrebbe essere eseguita a distanza con tavolette grafiche e/o lettori di impronte digitali. Ricordiamo che il sistema che ha permesso il famoso errore sulla positività HIV di un paziente trapiantato all'ospedale Careggi di Firenze è originato dalla trascrizione manuale e non elettronica del dato. Poiché ogni atto compiuto (compilazione del diario clinico, prescrizione terapeutica, manovra chirurgica ecc.) comporta il riconoscimento di identità di chi lo compie tramite l'apposizione della firma o sua sigla, ogni medico quotidianamente firma carta, tanta carta. Attualmente molte organizzazioni (autonoleggi, catene alberghiere ecc.) permettono di firmare la carta di credito tramite una tavoletta elettronica, in sostituzione del comune scontrino fiscale. Persino nelle sagre di paese l'uso del palmare ha permesso di semplificare le ordinazioni ai tavoli, snellendo l'organizzazione del servizio e garantendo sempre la corretta identificazione dell'operatore, del cliente e del contenuto.

In Italia, stante la continua significativa riduzione di 
medici, nonché di infermieri sempre più insufficienti, qualcuno si chiederà: può la ICT migliorare il modo di lavorare dei sanitari rendendolo più efficace, efficiente e sicuro? Può la ICT eliminare ripetitività, burocrazia e ridondanza facilitando così una maggior dedizione alla cura del paziente? Progetti limitati ed isolati (braccialetto per la terapia, cartella clinica singola ecc.) non hanno apportato alcun reale vantaggio al modo di lavorare dei sanitari e sono destinati ad aggravare sistemi farraginosi e inefficienti.

\section{Un sistema che renda possibile l'analisi degli esiti clinici}

L'utilizzo della ICT, come valido ausilio per l'analisi clinica, è fortemente correlata alla valutazione di qualità professionale. I due parametri sono quasi sinonimi e sicuramente possono essere affrontati insieme.

Abbiamo già ricordato che un sistema informatizzato potrebbe essere in grado di valutare l'andamento clinico di un paziente. È esperienza comune, soprattutto nelle malattie croniche, che sistemi atti a fornire il trend dei valori di laboratorio di un paziente, possono farne meglio comprendere anche l'andamento clinico. Conoscere il valore attuale della clearance della creatinina di un paziente è certamente importante, ma ancor più utile è conoscere quanto e con quale velocità questo valore si modifica nel tempo: calo, crescita, stabilità? Accenniamo solo al fatto che molti sistemi computerizzati di analisi della CKD si basano sulla velocità di progressione della insufficienza renale cronica (4). Dopo un primo inquadramento diagnostico e terapeutico da parte del nefrologo, questi sistemi di analisi supportano il medico di base nella scelta di continuare a seguire in primis il paziente al permanere della stabilità della funzione renale o di affidarlo alle cure dello specialista in caso di progressione della malattia $(5,6)$. La famosa raccomandazione delle $\mathrm{K} / \mathrm{DIGO}$ di osservare il valore di almeno due creatininemie in sei mesi ha proprio questo compito (7). Lo stesso compito hanno oramai anche le cartelle cliniche "on line", come quella di Google Health (8) che permette di colloquiare con le cartelle delle più famose assicurazioni americane. È interessante in Google Health la possibilità di consultare dosaggi, effetti collaterali e controindicazioni di ciascun farmaco inserito nella terapia.

Accenniamo solamente, per la complessità di trattazione, a come la ICT possa essere strumento di aiuto a un professionista (non solo sanitario) a prendere decisioni (9). I sistemi esperti sono la punta di diamante della moderna ICT ed è innegabile che sistemi esperti di diagnosi e terapia siano ormai alla portata di tutti e molti disponibili anche sugli smartphone (il programma
iSthescope per Apple iPhone, che promette di sostituire il fonendoscopio classico, è stato scaricato da 500.000 utenti in solo 2 giorni!).

\section{Un sistema che aiuti a valutare il nostro operato e a prendere decisioni}

Gli indici di valutazione dell'attività di un reparto di degenza sono attualmente (e spesso solamente) l'analisi della degenza media, il turnover dei ricoveri, la spesa e/o il costo ed i DRG prodotti. Ma il livello qualitativo di attività di un reparto lo si valuta anche e soprattutto analizzando i risultati clinici ottenuti che, a seconda della complessità clinica del paziente, possono comportare degenze più lunghe e oneri più corposi. I tradizionali indici di valutazione non consentono di valorizzare il diverso impegno assistenziale rivolto a pazienti con comorbidità totalmente differenti. In sanità attualmente l'unico strumento di valutazione professionale è rappresentato dall'Audit. L'Audit clinico viene così definito: «Revisione retrospettiva della pratica professionale, con obiettivo di individuare le opportunità di miglioramento e di introdurle nella pratica professionale, che si basa sull'esame attento dei dati raccolti nella pratica clinica routinaria tratti dalla documentazione clinica, volta a valutare in modo volontario le azioni compiute in contesti e tempi determinati, allo scopo di identificare aspetti passibili di miglioramento in termini di efficienza ed efficacia» (10). Esempi di Audit clinici sono la valutazione del target del P (11) o dell'emoglobina in un gruppo di pazienti valutando e indagando le cause del mancato raggiungimento nel gruppo negativo. Di solito il target clinico viene indicato in percentuale; in altre parole quanti pazienti raggiungono il target. In questo caso la ICT è fondamentale per raggruppare i dati di più pazienti. Nella definizione stessa di Audit abbiamo visto, essere compreso il concetto di Revisione retrospettiva. $\mathrm{E}$ anche in questo caso una cartella clinica computerizzata che non renda possibile la visualizzazione trasversale dei pazienti o di analizzare i trend, mostra tutti i suoi limiti. Il sistema dell'Audit clinico è adottato anche da JCI che, per certificare la qualità di un processo assistenziale, necessita di almeno due indicatori clinici desunti dalla linee guida valutabili in TUTTI i pazienti trattati (12). In altre parole, guardando ad esempio alla terapia dell'anemia, sarebbe fondamentale conoscere quanti pazienti presentano valori di emoglobina superiori a $13 \mathrm{gr} / \mathrm{dL}$, tra 12 e 13 , tra 12 e 11, tra 10 e 11 , sotto $10 \ldots$ ecc. In rete si trovano esempi di questi piccoli ausili ma rimane purtroppo ineluttabile la necessità di inserimento manualmente dei dati (13). I dati del censimento SIN 2009 (i provvisori sono stati presentati a Roma in occasione di NexGeneration) 
mostrano che il $63 \%$ dei centri trascrive manualmente il risultato degli esami di laboratorio e che spesso tale compito spetta al medico. Questa pratica è assolutamente risibile, anche alla luce dei dati di CERGAS-Bocconi pubblicati nel numero 3 del GIN 2010, che evidenziano come i nefrologi siano in netto calo con un turnover assolutamente penalizzante. Ebbene, come non pensare che questa attività di copiatura conduce a perdita di tempo? Il tempo di un nefrologo sta cominciando a diventare ancor più prezioso ed è opportuno che quello disponibile sia impiegato per dare il massimo del supporto culturale e clinico assistenziale, riducendo i tempi morti e le attività a basso valore aggiunto. I medici e gli infermieri, per altro, non hanno frequentato corsi di bella calligrafia nel loro percorso di studio e tanto meno hanno trascorso periodi di ritiro conventuale dediti alla copiatura di manoscritti. Si sente dire che la copiatura manuale degli esami di laboratorio, permette di valutare subito i problemi del paziente e fornisce un'ordinata stesura dei dati quando, ad esempio, si viene chiamati per consulenza al letto del malato. Basterebbe aver avuto esperienze di teoria degli errori o aver frequentato le sale controllo della ENAC (controllori di volo) per capire che la copiatura rende assuefatti e poco attenti oltre, ad essere foriera di errori. Questo è tanto vero che in alcune aziende ospedaliere tale pratica è stata vietata, senza però essere stata sostituita da sistemi che permettono di fruire di un immediato e facile accesso ai dati!

L'auspicio è che quindi i sistemi di ICT in Sanità amplino e di molto il loro campo di applicazione senza perdere d'occhio lo scopo principale: porre veramente al centro il paziente ed i suoi problemi.

Infine sarebbe anche opportuno che gli utenti finali di un sistema (medici e infermieri), vengano coinvolti nell'ideazione e stesura delle diverse fasi di progettazione. Il coinvolgimento di chi, fra gli utenti, ha maggior dimestichezza con l'informatica e consapevolezza di quello che è possibile fare ed ottenere (magari anche con personali esperienze di qualche applicativo), permetterebbe di ottenere dei sistemi informatici caratterizzati da maggior semplicità di utilizzo, davvero dotati della possibilità di una completa fruizione di informazioni e dati.

$\mathrm{Ci}$ piace concludere con una frase di Marchionne al Meeting di Rimini 2010 che, comunque la si pensi, può offrire un serio spunto di riflessione per comprendere quanto abbiamo cercato di affermare in questo articolo: «Fino a quando non ci lasceremo alle spalle $i$ vecchi schemi non ci sarà spazio per vedere nuovi orizzonti».
Indirizzo degli Autori:

Giuseppe Quintaliani, MD

Via Francesco Maturanzio 31

06124 Perugia

quintaliani@yahoo.it

\section{Bibliografia}

1. Sole24 Ore Sanità, 3-9 febbraio 2009.

2. l'Information\& Comunication technology: http:// it.wikipedia.org/wiki/Information_and_Communication_ Technology

3. Losito A, Raimondi C, Zampi I, Quintaliani G, Lorusso L. Diagnosi ed analisi computerizzata delle variazioni della funzione renale Giornale Italiano di Nefrologia 1985; 2: 179-80.

4. CKD. Jones C, Roderick P, Harris S, Rogerson M. An evaluation of a shared primary and secondary care nephrology service for managing patients with moderate to advanced CKD. Am J Kidney Dis 2006; 47(1): 103-14.

5. Kinchen KS, Sadler J, Fink N, Brookmeyer R, Klag MJ, Levey AS, Powe NR.The timing of specialist evaluation in chronic kidney disease and mortality. Ann Intern Med 2002; 137(6): 479-86.

6. Stevens PE, Farmer CK, Hallan SI. The primary care physician: nephrology interface for the identification and treatment of chronic kidney J Nephrol 2010; 23: 23-32.

7. National Kidney Foundation SG: K/DOQI clinical practice guide- lines for chronic kidney disease: evaluation, classification, and stratification. Am J Kidney Dis 2002, 39(Suppl 1): S1-266.

8. https://www.google.com/accounts/ServiceLogin?service= health\&nui $=1 \&$ continue $=\mathrm{https} \%$

9. $\mathrm{A} \% 2 \mathrm{~F} \% 2 \mathrm{Fhealth}$.google.com $\% 2 \mathrm{Fhealth} \% 2 \mathrm{Fp} \%$ $2 \mathrm{~F} \&$ followup=https\%3A\%2F\%2Fhealth.google. com\%2Fhealth\%2Fp\%2F\&rm=hide

10. de Lusignan S, Chan T, Stevens P, et al. Identifying patients with chronic kidney disease from general practice computer records. Family Practice 2005; 22: 234-41.

11. Glossario regione Toscana febbraio $2000 \mathrm{n}^{\circ} 30$.

12. MereuMC, Bolasco PG, et al. Il trattamento dell'osteodistrofia nei pazienti uremici in dialisi: risultati del primo Audit sardo. Giornale Italiano di Nefrologia 2004; 21(4): 362-70.

13. Giuseppe Quintaliani et al: Chronic kidney disease certification process manual by the Italian Society of Nephrology (SIN) J Neprol 2009; 22: 565-570

14. http://www.renalgate.it/formule.htm 